\title{
FÚTBOL Y PATRIOTISMO EN AMÉRICA DEL SUR: ENTRE LA ÉLITE Y LA SOCIEDAD CIVIL
}

\section{FOOTBALL AND PATRIOTISM IN SOUTH AMERICA: BETWEEN THE ELITE AND CIVIL SOCIETY}

\author{
Ekain Rojo-Labaien \\ Universidad del País Vasco UPV/EHU \\ Ekain.Rojo@ehu.eus
}

Resumen: El fútbol se erigió en un pilar comunitario en el siglo XX en las sociedades de América del Sur, durante el proceso de construcción nacional caracterizado por las desigualdades sociales. En esta coyuntura, su dimensión patriótica originaria ha sido apropiada distintivamente, tanto por la élite como por la sociedad civil. Este artículo aborda, mediante el análisis histórico del Mundial de Argentina 1978, el Mundialito de Uruguay 1980 , y las protestas sucedidas durante la Copa de Confederaciones de 2013 en Brasil, tanto la instrumentalización hegemónica del evento del fútbol por parte de la élite en el poder como el uso contra hegemónico de los ciudadanos. Las conclusiones permiten desterrar así las visiones unidireccionales sobre las movilizaciones deportivas de condición patriótica, y realzar también la evolución transformadora acaecida en el evento del fútbol. Asimismo, el estudio sociológico del deporte emplazado en América del Sur permite vehicular y extrapolar el carácter conflictual de las sociedades actuales.

Palabras clave: Fútbol, América del Sur, nacionalismo, sociedad civil, funcionalismo, cambio social, dictadura

\begin{abstract}
Football in South American societies became a community pillar over the twentieth century, against the backdrop of nation building processes characterized by social inequalities. In this context, both elites and civil society made use of football's original patriotic influence in distinctive ways. Thus, through a historical analysis of three sporting events - the 1978 World Cup in Argentina, the 1980 'Mundialito' in Uruguay, and the protests that took place during the 2013 Confederations Cup in Brazil - this article looks at the different ways in which football is used by the ruling governments and the citizens; namely a hegemonic use by the elite and a contra-hegemonic usage by the civil society. In this manner, the conclusions allow to banish the persistent beliefs about the one-sided hegemonic character of the sport phenomenon and highlight the transformative evolution reflected in football events. Similarly, the sociological approach to sport in South America leads to conclude the conflictual nature of contemporary societies.
\end{abstract}

Key words: Football, South America, nationalism, civil society, functionalism, social change, dictatorship 


\section{Introducción}

Las proclamas y protestas sucedidas en numerosas ciudades de Brasil, en un país emergente de América del Sur, en torno a la acogida y a la organización de la Copa de Confederaciones y el Mundial de fútbol en 2013 y en 2014, suscitaron un análisis social que contravenía la visión de la realidad sobre el continente americano y el fútbol, y que lo circunscribía perpetuamente a ser un artilugio de manejo de las masas al servicio de la élite en el poder. Así, este artículo persigue acometer una investigación primordialmente opuesta, que englobe el conflicto ineludible en las sociedades contemporáneas, la dialéctica propia de cualquier hecho social, y que el acontecimiento del fútbol permite vehicular (Bromberger, 1995: 377).

El análisis persigue, por ende, discernir la esencia del evento del fútbol en lo que concierne a su utilización por el poder político y también a la iniciativa emancipada de los pueblos supeditados de los estados. Con ese fin, se erige en un análisis empírico y evolutivo que aúna el Mundial de 1978, el Mundialito de 1980 y la Copa de Confederaciones de 2013. Los primeros dos casos temáticos son paradigmas de la concepción del alcance patriótico del fútbol a beneficio de los gobiernos totalitarios. Por otra parte, el evento brasileño más reciente esgrime la bifurcación del fútbol entre su vertiente hegemónica a manos de la élite, y el ámbito de resistencia en su utilidad por parte de la sociedad civil. El estudio no engloba el Mundial de 2014 en Brasil habida cuenta que el uso contra hegemónico del deporte en la Copa de Confederaciones de 2013 no tuvo una continuación similar en la sociedad brasileña. La extrapolación consecuente de las nociones diversas divisadas en América del Sur propicia una visión amplia de la significación del fútbol en la era contemporánea, que supere las reflexiones unidireccionales existentes en torno al deporte (Boyle y Haynes, 2009: 151).

\section{Metodología}

Así las cosas, el estudio busca vislumbrar mediante una metodología histórica y un marco teórico emplazado en el estudio sociológico del deporte, la diversa influencia comunitaria del acontecimiento del fútbol en América del Sur. Entre los tres arquetipos de estudio elegidos para el análisis empírico figuran el Mundial de 1978, bajo el auspicio de la dictadura de Videla, y el campeonato singular nombrado Mundialito, disputado en Uruguay en 1980 y albergado por un régimen dictatorial de la misma índole. En estos dos casos, el acercamiento analítico a partir de las ciencias sociales se ha constituido tradicionalmente a partir de una certeza previamente funcionalista, hegemónica y unidireccional de las sociedades contemporáneas, y que confinaba al acontecimiento del fútbol a conceder únicamente un ámbito instrumental para el poder instituido (Manzenreiter, 2006 : 149). La corriente de pensamiento funcionalista propugna que el fútbol, efectivamente, lleva a cabo una función para el correcto engranaje de los estados, a saber, la de educar a los ciudadanos en los valores hegemónicos de cada sociedad y propiciar la identificación con la nación mediante los partidos internacionales (Jarvie y Maguire, 1994: 9-10). En otras palabras, enfatiza en la utilidad alienadora del evento para la constitución homogeneizadora inherente del estado-nación (Goldberg, 2002: 140). No obstante, este acercamiento monolítico no contempla la diversidad y la pugna de fuerzas contradictorias existentes en la sociedad, y divulgadas en el ámbito del fútbol mediatizado (Billings, 2008: 16). En esta coyuntura analítica, el tercer caso de estudio de la Copa de Confederaciones 2013 acogida en Brasil y su posterior desarrollo en la sociedad, se emplaza como paradig- 
ma de la instrumentalización diversa y de múltiples vertientes del acontecimiento del fútbol en el siglo $\mathrm{XXI}$.

En este sentido, se puede aseverar que el acercamiento de las ciencias sociales ha traspasado el umbral pasado que relegaba al deporte a una dimensión despreciativa al servicio únicamente del control de las masas. Sin embargo, la pervivencia de una visión hegemónica en la opinión pública vertida sobre el fútbol y la sociedad justifica la pertinencia de este estudio sobre la fisonomía del deporte.

\section{Marco teórico y contextualización histórica}

En palabras del escritor Eduardo Galeano fallecido en el año 2015, el fútbol y el ideario de patria siempre estuvieron ligados en América del Sur, y los políticos y los dictadores que condicionaron su historia contemporánea trataron de obtener réditos de esa unión identitaria (Galeano, 2003: 38). Joseph L. Arbena relativiza la aportación del fútbol a las comunidades nacionales de Sudamérica, pero añadiendo que cuanto menos este deporte se fue coaligando con diversas iniciativas sociales, políticas y económicas para facilitar la construcción nacional (Arbena, 1996: 233). El proceso de nacionalización en América de Sur durante el siglo XX fue caracterizado por la inestabilidad política y por la desigualdad social. Por consiguiente, la esencia patriótica del fútbol supuso una vía de escape originada por los diversos gobiernos para tratar de alejar a sus ciudadanos de las reivindicaciones políticas y sociales las cuales pudieran cuestionar su hegemonía (Brohm y Perelman, 2006). No obstante, compartiendo el razonamiento de Marc Auge, entre otros, su alcance no se limita a ser un artefacto del poder establecido. El evento futbolístico, como demuestra la realidad poliédrica en América del Sur, posee una identidad doble y complementaria que lo convierte, a su vez, en opio del pueblo y en instrumento para el cambio político (Auge, 1999: 59, 61).

El ascendiente polifacético del deporte radica en gran medida en el hecho de que el fútbol se engendró desde sus inicios como un ancla de las identidades colectivas ante la deriva individualista de las sociedades industriales (Wahl, 1997: 51). A saber, la instauración del evento deportivo, y su razón de ser, precedió a las posteriores utilizaciones patrióticas del acontecimiento, siendo éstas de distinta índole. En palabras de Eric Dunning, la demanda del deporte representativo es inherente a la modernidad, ya que la falta de unificación nacional y la ausencia de medios de transporte y comunicación de las formas societales preindustriales imposibilitaban su aparición anterior (Dunning, 1992: 264). Del mismo modo, el carácter reglamentado y competitivo de los deportes modernos, reflejos de los ideales del mundo capitalista, los diferenciaban de los juegos provenientes del Medievo (Darbon, 2008: 1). Los deportes se extendieron a través de la globalización como símbolo de los valores de la civilización occidental encarnados por la homogeneización de los estados-nación y el libre mercado (Salvador, 2004: 25). A este respecto, como sostiene Christian Bromberger (1998 : 59), el fútbol se erigió en un espacio consustancial de la representación y la diferenciación de la identidades colectivas mediante confrontaciones miméticas, radicando ahí en gran medida su eclosión decimonónica en la sociedad y su extensión casi planetaria.

En este contexto, el alcance del denominado imperio informal británico, que trascendía su ámbito territorial político, propició la adopción y la eclosión del fútbol inglés en América del Sur en el transcurso del siglo XIX al siglo XX. En efecto, el deporte sobrepasó nítidamente los confines del imperio británico a medida que la industrialización 
británica se desplegaba por todo el mundo. El fútbol se estableció primeramente en los llamados laboratorios de la modernidad, como sucedió precozmente en América del Sur, es decir, en metrópolis, puertos y en núcleos industriales florecientes, y fue extendido a través de la construcción de las vías de comunicación interiores, como la línea del ferrocarril (Dietschy, 2010: 89). Considerando la argumentación del antropólogo Sebastien Darbon, no es conveniente vislumbrar en la transmisión del fútbol un propósito preconcebido por parte del imperio británico para extender su dominación en el mundo, ya que los emigrantes británicos no pretendían compartir su actividad de ocio con los oriundos del país concernido (Darbon, 2008: 306). En cualquier caso, esa apreciación no conlleva que la hegemonía británica por medio del fútbol no fuese notoria. Efectivamente, en las primeras décadas del siglo XX, las élites locales se unieron a los expatriados europeos en la disputa del fútbol en espacios segregados de grandes urbes, prosperas e industriales, americanas, tales como Buenos Aires, Montevideo, Santiago, Sao Paulo o Río de Janeiro (Natali, 2007: 268).

Joseph L. Arbena resalta la faceta irónica que en América del Sur a medida que los sentimientos patrióticos se agrandaban en el ocaso del siglo XIX, los promotores del nacionalismo que buscaban la diferenciación con las metrópolis europeas, imitaban la formula de organización y representación política de esos pretéritos dominadores (Arbena, 1996: 222). Utilizaron así precozmente, los deportes de esencia europea, dejando de lado el amplio legado cultural y deportivo propio, para generar espacios de pertenencia a la comunidad. Buscaban legitimar su construcción del modelo del estado-nación originado en Europa y dibujado en las líneas territoriales que habían forjado los colonizadores. La evolución posterior en el siglo XX agudizó esa tendencia creolizadora y propició que el fútbol se convirtiese en eje central de expresión colectiva. En palabras de Ezequiel Fernández Moores (2006: 67), sobre todo en las realidades de Brasil y Argentina, el fútbol propició desafiar en un ámbito simbólico y representativo la superioridad ejercida por los europeos, habiendo transcurrido décadas desde el fin de la supeditación colonial. De ese modo, permitió empequeñecer, las crisis identitarias en la oposición con un antagonista exterior. Los estados requerían símbolos y tradiciones para establecer una nación que aglutinase a sus ciudadanos, y los identificase entre si. En esta coyuntura, a partir de los años 1920, el discurso nacional de las sociedades de América del Sur se instituyó en gran medida en el marco del fútbol (Alabarces y Rodríguez, 1999: 121, 122). En Argentina se erigió el estilo de juego criollo como baluarte de diferenciación nacionalista, y promovido por el diario El Gráfico. El modo de jugar al fútbol de los argentinos se instituyó como un reflejo de los rasgos genuinos de una sociedad heredada de sus antepasados europeos, pero moldeada por las condiciones propias del territorio (Darbon, 2008: 270-271). Ese atributo se perpetuó en el siglo XX como un bastión identitario argentino (Roldán, 2006: 146).

En cualquier caso, el modelo de construcción nacional de los países de América del Sur que se reflejaba a través del idioma del fútbol (Gordon y Helal, 2001: 142), se basó en el conflicto social y estaba caracterizado por el sometimiento de las clases y de los grupos étnicos más desfavorecidos, es decir, de los negros y de los mestizos (Natali, 2007: 269), como sucediera en el transcurso del primer tercio del siglo XX en el fútbol de Brasil. La adopción del deporte por la sociedad favoreció finalmente que el fútbol se convirtiese en un adalid de la integración en la nacionalización de América del Sur. En 1930, Uruguay acogió la primera edición del Mundial, cuya organización resultó ser la prioridad para su gobierno al coincidir con la efeméride del centenario de la gestación del estado en 1830. La representación uruguaya se consagró campeona, y así, completó el acontecimiento nacional ideado por el estado 
ávido de símbolos (Boniface, 2002: 31). Posteriormente, el mandato peronista entre 1946 y 1955 en Argentina, impulsó el fútbol como vía de integración nacional asentado en la propaganda política y el control de las masas, a través de la dimensión patriótica del deporte (Duke y Crolley, 2001: 103-104).

La Guerra denominada del fútbol, breve conflicto generado entre El Salvador y Honduras en 1969, tuvo su origen en un cúmulo de factores de confrontación enconada, y un marco internacional proclive a la guerra (Rouquié, 1971: 1315). Se desencadenó inmediatamente después de la eliminatoria clasificatoria para el Mundial de 1970 entre las representaciones nacionales de El Salvador y Honduras, y de ahí se derivó la confusa definición para la historia de Guerra del fútbol. En realidad, ante todo, el fútbol había sido usado, premeditadamente, para exacerbar las pasiones nacionalistas, en palabras de Ryszard Kapuściński (1992: 215), tan necesarias para poder desencadenar la guerra y fortalecer así el poder de las oligarquías en los dos países. La Guerra del fútbol supuso el sometimiento del fútbol a la funcionalidad requerida por las elites directoras de los respectivos países. En efecto, a medida que se erige en una forma cultural fundamentada en la competición, el fútbol se presta singularmente a las manipulaciones políticas e ideológicas (Boyle y Haynes, 2009: 147). No obstante, tal y como acentúa Jeremy MacClancy, los súbditos también pueden emplear su organización con destreza a fin de acometer sus objetivos, a saber, enfrentarse al control y reivindicar sus valores distintos (MacClancy, 1996: 11).

En palabras de Paul Yonnet (2008: 21), el deporte a través de la identificación ahuyentó el peligro de disolución social que atormentó a los ciudadanos desde el inicio del proceso de la individualización. El hito comunitario del evento deportivo trascendió así precozmente, a la manipulación monopolística del poder establecido. En este ámbito, es pertinente trasladar al espectro deportivo la crítica esgrimida por Armand $y$ Michèle Mattelart al pensamiento marxista, y que denominan con el funcionalismo de izquierdas (1987: 37). Es decir, los acercamientos extendidos en el pensamiento marxista sobre los medios de comunicación y la competición deportiva disponen de un juicio crítico, pero perduran en la visión gestada por el funcionalismo. Jesús Martín Barbero (1980), siguiendo la línea argumental de los Mattelart, acusa a la corriente crítica en las ciencias sociales de estar atrapada en la lógica funcionalista: "Lo que no cabe definitivamente en ese modelo es la contradicción y el conflicto". Por el contrario, la investigación empírica en la que se basa este artículo, situada en América del Sur, pretende resaltar la dialéctica inherente en el fútbol y en la sociedad, con un método histórico y comparativo propio de las ciencias sociales y que posibilita conclusiones generalizables (Sonntag, 2010: 209).

\section{Discusión de datos}

\subsection{El Mundial 1978 en Argentina unido a la construcción política de la Junta Militar}

El énfasis de la influencia patriótica del deporte como método de control social fue antecedido en Argentina por el régimen del general Agustín Pedro Justo en los años 1930. Posteriormente, Juan Domingo Perón empleó el deporte tras el fin de la Segunda Guerra Mundial a modo de método para inculcar disciplina y patriotismo en la sociedad y equiparándose con la visión promulgada por el fascismo de Benito Mussolini en Italia (Dietschy, 2010: 253). Consecuentemente, el deporte, y notablemente el fútbol, habían sido constituidos en baluartes hegemónicos para las elites en el poder en Argentina. En gran medida, el fútbol había sido erigido en un rito nacional 
a raíz de la necesidad de fraguar un pilar de identidad (Archetti, 1994: 229), y había eclosionado en el siglo XX en la coyuntura de la crisis de identidad del estado argentino (Alabarces, 1999: 78). La estructuración simbólica del estado-nación en torno al evento del fútbol en la coyuntura compleja de la construcción nacional en Argentina, fue apropiada por los grupos dirigentes de carácter autoritario para ejercer su dominio. La dictadura militar de Jorge Videla fue de esta manera, un eslabón más en la cadena histórica del estado argentino, cuya acción de gobierno tuvo en el epicentro, y culminación del manejo patriótico del fútbol en el país, la organización del Mundial de 1978. El hito ha sido ligado negativamente en la historia general, y en Argentina en particular, a la dictadura y a los crímenes auspiciados en ella.

La dictadura militar de Jorge Videla se instauró en el poder de Argentina en el 24 de marzo de 1976, tras efectuar un golpe de estado en un contexto de confrontación entre grupos de izquierdas y grupos paramilitares impulsados por el gobierno. En esta coyuntura, el régimen se erigía a modo de último bastión para restablecer la "paz social" en el país (Roldán, 2006: 131). El marco en el que la dictadura emprendió su andadura y el que el país se acercó a la acogida del Mundial, fue el de un asesinato de motivación política cada cinco horas y el de la explosión de una bomba cada tres horas. La inflación como reflejo de la economía, alcanzaba por su parte la cifra record del 56\% en marzo de 1976 (Archetti, 2006: 135). La dictadura tuvo como finalidad primordial y razón de ser desterrar la amenaza de los grupos de izquierdas revolucionarios, y en su ejercicio de poder, hasta 1983, la represión, el secuestro y el asesinato fueron medios rutinarios alcanzando la cifra de 30.000 asesinados (Smith, 2002: 70). El Mundial de fútbol obtendría por parte del gobierno una atención principal, después que la inacción o imposibilidad en este sentido, del anterior gobierno peronista - comandado por Isabel Perón tras el fallecimiento de su marido Juan Domingo Perón en el año 1974 - hiciese sopesar que Argentina finalmente no conseguiría acometer a tiempo los preparativos para acoger el campeonato mundial de fútbol. En palabras de Diego P. Roldán (2006: 132), la asociación entre el evento del fútbol y la nación, y en consecuencia, la organización del evento internacional, resultaba una pieza angular a fin de perpetuar los valores del régimen en la sociedad. Roldán denomina, desde un prisma de manejo de los ciudadanos, que "los fascinantes narcóticos del consenso comenzaron a ser distribuidos a través de las redes de poder". El esmero de la autoridad de Videla por el fútbol, y la magnitud del evento corroboran nítidamente la búsqueda de ese objetivo a través del deporte.

La Junta Militar mejoró la infraestructura audiovisual e implementó la televisión a color. Empleó el $10 \%$ del presupuesto anual del estado a la organización del Mundial. Haciendo una comparativa, en el Mundial de 1982 acogido en España, y con el evento del fútbol todavía más extendido en el mundo, el gobierno español empleó el tercio del presupuesto utilizado por su predecesor. Igualmente, el dinero invertido por Argentina para la organización en 1978 supuso el $40 \%$ del montante que el gobierno dedicaba anualmente a la educación (Rein y Davidi, 2009: 674-675). Videla había equiparado así el modelo de organización de Benito Mussolini concerniente a la acogida del Mundial de Italia en 1934, cuando el dictador italiano no dudó en realizar una notable inversión a pesar de la crisis económica que azotaba Italia y al resto del mundo occidental tras el año 1929. Incluso hay fundadas sospechas que, a imagen del gobierno fascista de Italia, los militares sobornaron a la selección de Perú para que permitiese una holgada victoria de Argentina en la fase de clasificación de 1978, en aras de posibilitar el triunfo final de la selección nacional (Duke y Crolley, 2001: 113).

La Junta Militar trató de reivindicarse mostrando en el marco internacional que Argentina era capaz, bajo su mando, de albergar convenientemente uno de los mayores 
eventos del mundo. El militar responsable de la comisión del evento, Antonio Merlo, declaró en el periódico Somos que el objetivo primordial era resaltar la competencia del país para albergar el Mundial (Archetti, 2006: 137). La razón de ser del campeonato, a su vez, radicaba en ocultar la violencia del estado en el éxito organizativo y en la relevancia mundial del fútbol, ya en constante crecimiento. Meses antes de comenzar el Mundial la dictadura intensificó la represión contra los grupos de izquierdas y alejó a los más desfavorecidos de las ciudades, a fin de construir una imagen artificial de unión pacífica y de bienestar del país. El evento estuvo legitimado, y por ende la dictadura militar, por la asistencia al campeonato de 15 selecciones representantes de estados de estructura o ideario democrático. La sola sospecha que a pocos metros de los estadios se practicaba sistemáticamente la tortura, no alteró el orden previsto por los organizadores internacionales del acontecimiento. La visión generalizada de las autoridades políticas mundiales fue que, contradiciendo las iniciativas de grupos como Amnistía Internacional, era necesario limitar el fútbol en el espacio de la sociedad civil, independiente de la iniciativa de las políticas de estado. Repetían el discurso manido de diferenciar la política y el deporte (Archetti, 2006: 135-136). Se retrotraía, en consecuencia, al debate del amateurismo y a la independencia del deporte que propugnaba el Reino Unido ante la utilización política del deporte de la Italia fascista y la Alemania nazi en los años 1930. En esta coyuntura, el gobierno británico tuvo que claudicar ante el manejo político de esos dos regímenes (Beck, 2001: 248; Holt, 2002: 80). El efecto en el ámbito internacional fue similar en el transcurso del Mundial de 1978.

El gobierno de Videla obtuvo un auge organizativo que fue redondeado por la victoria de la selección nacional argentina. Sin embargo, el empleo patriótico del fútbol durante el Mundial de 1978 no fue unidireccional. La originaria asociación entre fútbol y nación en América del Sur, posibilitó que, los prisioneros políticos, habiendo sufrido torturas, y pese a temer por sus vidas en las celdas donde estaban recluidos, celebraran también la victoria de la selección nacional que ansiaba Videla. Alfredo Pérez Esquivel, galardonado posteriormente en 1980 con el Premio Nóbel de la Paz, dilucidó la aparente contradicción que recreaba el evento del fútbol unido con la eclosión de patriotismo.

En la cárcel, como los guardias también querían escuchar los partidos, el relato radial nos llegaba por altoparlantes. Era extraño, pero en un grito de gol nos uníamos los guardias y los prisioneros. Me da la sensación de que en ese momento, por encima de la situación que vivíamos, estaba el sentimiento por Argentina." (cfr. Agüero Wagner, 2010)

El hecho de que el campeonato hubiese sido promovido por el régimen que los reprimía, no eludió la celebración nacionalista de los reprimidos en torno a la victoria de su representación nacional. Sería contraproducente concluir de ello el logro total del uso del deporte como opio del pueblo. En realidad, este artículo sostiene que la celebración patriótica en torno al fútbol dictaminado y dirigido por las elites, no tiene una traslación absoluta en la iniciativa de la sociedad. El ideario funcionalista unilineal se resquebraja a la luz de diferentes extremos del fútbol en América del Sur. Los prisioneros políticos antepondrían su apego al país a la manipulación emprendida por la Junta Militar a través del mismo conducto, el fútbol y la euforia nacional impregnada en la sociedad. Cuando en el año 1983 se restableció el sistema democrático en Argentina, el equipo nacional y el seleccionador Cesar Luís Menotti, fueron requeridos a explicar su actuación deportiva a favor del régimen. Los jugadores y el entrenador declararon que representaban a la gente y que jugaron para la gente (Archetti, 2006: 143), con independencia del gobierno en el poder. 
Sin embargo, no es menos cierto que aquella primeriza victoria de la selección nacional argentina en un Mundial quedó en la memoria colectiva del país como motivo de vergüenza y pesar, dado el esclarecimiento posterior de los crímenes cometidos por la Junta Militar en ese periodo. El segundo Mundial logrado por Argentina en la edición de 1986 acrecentó además esa connotación negativa, dado que en esa nueva coyuntura el equipo liderado por Diego Armando Maradona logró la victoria, batiendo, entre otros, a la selección de Inglaterra. Había tomado la revancha, en un ambiente marcadamente nacionalista, de la derrota reciente sufrida en las Islas Malvinas por la armada argentina frente a la armada homóloga británica, y que había supuesto el desmoronamiento de la dictadura militar del general Videla. Así las cosas, el Mundial de Argentina de 1978 resulta un signo clarificador y reciente de la utilización diáfana del poder establecido para servirse del carácter patriótico del deporte como una fuente de legitimación sobre sus ciudadanos, y en la esfera internacional. No obstante, el campeonato argentino y, el artículo en la globalidad, evidencian los límites de su uso hegemónico para alienar a la sociedad.

El evento del fútbol refleja las pulsiones diversas albergadas en las sociedades contemporáneas, y que engloban igualmente los marcos de resistencia de la iniciativa popular. De esta manera, cabe resaltar que la dictadura del general Videla no consiguió apoderarse totalmente de la condición patriótica unida al fútbol, ya que no pudo alcanzar el sometimiento integro de los argentinos a su causa (Eisenberg et al., 2004: 289). Diego P. Roldán (2006: 146) sostiene que a través del campeonato la dictadura obtuvo cuanto menos, "una adhesión pasiva o indiferencia momentánea" de la sociedad concerniente al programa de asesinatos del estado. Con todo, es razonable extraer la conclusión que la asociación originaria entre el fútbol y la comunidad nacional en Argentina propició en ese contexto que prevaleciese el alcance nacional a la toma de posición colectiva sobre la represión gestada desde el poder. A este respecto, la repulsa existente en la actualidad en Argentina sobre el Mundial de 1978, dado su ligazón en la memoria colectiva con el régimen de Videla, reflejaría la condena de los ciudadanos a la manipulación dirigida por la dictadura de la primera consecución mundial de la representación argentina de fútbol.

\subsection{El Mundialito inédito de Uruguay 1980 como signo contra hegemónico en el fútbol}

Si el Mundial de Argentina fue un acontecimiento que no permite aventurarse sobre el éxito final e irreflexivo de la manipulación del fútbol por la clase dirigente, el campeonato contiguo de carácter extraordinario acogido en Uruguay, entre diciembre de 1980 y enero de 1981, propicia acometer una parecida argumentación. A saber, fomenta la reflexión de que el acercamiento funcionalista en torno al fútbol en el espacio de las ciencias sociales, y fuertemente extendido en la sociedad, es analíticamente limitado. La dictadura instaurada en 1973 perseguía perpetuarse en el poder con el impuso patriótico generado en la sociedad por el fútbol. Con esa finalidad, logró el beneplácito de la FIFA para albergar el primer campeonato mundial, denominado popularmente con el nombre de Mundialito, y que aglutinaría a las representaciones nacionales que hubiesen ganado alguna edición del Mundial en su historia. La sola excepción fue la selección de los País Bajos, que sustituyó al Reino Unido en el torneo, tras la negativa de esta representación para participar en el campeonato. La representación nacional uruguaya fue la vencedora del torneo. No obstante, el fútbol no proporcionó los efectos deseados al régimen.

En cualquier caso, la acogida del certamen internacional de fisonomía deportiva se encuadraba en la longeva tradición uruguaya anclada en la competición del fútbol. 
Ésta databa notablemente de la organización de la primera edición del Mundial en 1930 y ostentadora a partir de entonces de una inevitable vertiente nacionalista en el mundo. Uruguay se erigió en la primeriza potencia dominadora en el fútbol tras haber resultado vencedora en el marco de las competiciones de fútbol precedentes durante los Juegos Olímpicos disputados en la década de 1920. Posteriormente se había alzado victoriosa en el Mundial organizado a domicilio. Así, el fútbol posibilitó en gran medida a Uruguay de posicionar simbólicamente al país en el mundo, cuyo exiguo territorio resultaba ínfimo en la bifurcación entre las potencias vecinas de Argentina y Brasil (Galeano, 2003: 50). En esta coyuntura, el estilo de juego defensivo forjado en el fútbol, fue considerado una translación consecuente, inherentemente, de la identidad uruguaya, cuyo emplazamiento le obligaba a defenderse ante países de mayor envergadura (Archetti, 1994: 235). Con todo, tal y como sostiene Gustavo San Román (2005: 654, 655), el fútbol se convirtió tempranamente en enclave primordial de identidad y aglutinador de la nación uruguaya.

A semejanza del auge precoz en el fútbol, Uruguay se caracterizó notablemente por ser una de las democracias más duraderas en América del Sur, cuyo establecimiento fue coetáneo a la primera etapa del país como estado soberano. La democracia arraigada en un sistema de partidos fue únicamente interrumpida en el ocaso del siglo XIX, en la década de 1930 y, ante todo, durante la dictadura más longeva del estado entre 1973 y 1985 (Lanzaro, 2013: 241), y el que fue el gobierno más represivo (San Román, 2005: 640). Así las cosas, el hito autoritario en Uruguay coincidió premeditadamente con la organización del evento internacional denominado Mundialito en 1980. A diferencia de las dos potencias colindantes, e incluso englobando al territorio de América del Sur en la totalidad, Uruguay se señalaba por disponer una estabilidad singular en el siglo XX.

No obstante, la crisis económica y social que golpeaba al estado y a su sistema político en los años 1960 propició el caldo de cultivo finalmente para la instauración del régimen dictatorial con 12 años de duración (Manero, 2013: 6). Jaime Yaffé pone en relieve el binomio entre la vertiente militar y la cívica durante la dictadura, encarnada por la continuidad en los mandos políticos de los anteriores gobernantes democráticos, como fue el caso del presidente Juan María Bordaberry hasta el año 1976 y que fuera sustituido por otro estadista civil (Yaffé, 2012: 16-17). El régimen dictatorial argüía una continuidad en la línea política de Uruguay y trasladó como su razón de existir, y en analogía a la realidad contemporánea en la fronteriza Argentina, el sometimiento de los grupos revolucionarios de izquierdas. Con ese fin, compatibilizó el terrorismo de estado junto con políticas de propaganda intensas para legitimar su poder (Ibid., 20-21). En palabras de Daniel Corbo (2007: 32), la dictadura estuvo obsesionada en la legitimación e institucionalización de su autoridad.

En este punto, a semejanza de la dictadura militar dirigida en Brasil por Emilio Garrastazu Medici en 1970, durante el Mundial de México, y la comandada por Videla en el Mundial 1978 en Argentina, el régimen uruguayo trató de obtener réditos políticos de un mega evento deportivo internacional en aras de perpetuar su dictadura. Así, era partidario de intentar diluir el alcance en la sociedad de la represión contra los grupos de izquierdas en el espacio de la pasión política asociada con el acontecimiento del fútbol. En cualquier caso, la dictadura militar se mostró discordante al inicio sobre la acogida del Mundialito, ya que temía de la llegada de los periodistas internacionales a Uruguay que difundiesen la represión existente. Además, el régimen no se mostró tan proclive en el gasto a realizar como su homóloga argentina, y el certamen requirió de participación privada (Manero, 2013: 8, 9). Cuando el procedimiento de organiza- 
ción fue validado, la dictadura dispuso contiguamente en el tiempo dos eventos para que anclasen su autoridad en Uruguay.

El 30 de noviembre de 1980 se hizo el plebiscito de reforma constitucional que en caso de aceptación de la sociedad, extendía la autoridad de la dictadura, a raíz de la propuesta de profundos cambios constitucionales. El evento del fútbol un mes más tarde hubiera sido entonces el marco perfecto de celebración del régimen dictatorial tras el supuesto beneplácito de la sociedad en el referéndum. Había sido dirigido como medio a fin de neutralizar la sociedad civil (Corbo, 2007: 30). No obstante, sorpresivamente, habida cuenta que la consulta a la sociedad se encuadraba en un contexto de represión política, el referéndum escenificó la negativa de los uruguayos a la perpetuidad de la dictadura, con un total del $58 \%$ de los votos en contra. Así, el Mundialito dejaría de ser concebido como un marco de celebración completo para los dirigentes (Bednarik, 2010).

El deporte había sido el impulsor de la llegada de los receptores de la televisión a color al país. Bajo el auspicio de la FIFA, y en un tiempo caracterizado por la crisis y la represión llevada a cabo por el poder, el equipo nacional uruguayo se alzó finalmente con el Mundialito. Venció por 2-1 a la selección brasileña en la gran final. No obstante, más allá de la consecución deportiva, el resultado del evento deportivo no fue el ideado por la autoridad militar. El fútbol no cumplió el cometido programado, de la misma manera que el referéndum previo había tenido un resultado imprevisto para la dictadura. Dado que en este acontecimiento también se oponían intereses dispares, entre otros el anhelo hegemónico de la élite en el poder y el ansia de resistencia encarnado notablemente por los grupos de izquierdas sometidos y reprimidos por ella, el resultado se dirimió en una victoria amarga para la dictadura militar. Como explica Manero (2013: 12), hubo una pugna desigual entre el régimen en el poder y los presos políticos o militantes clandestinos por el uso del Mundialito como aparato propagandístico. Los militares disponían de los medios hegemónicos inherentes al ejercicio de la autoridad, mientras que los opositores hicieron uso del torneo a fin de socializar acciones de resistencia, tales como la propagación de mensajes contrarios a la dictadura y celebrando la victoria del no en el referéndum de reforma constitucional previo.

Un documental dirigido por Sebastián Bednarik (2010) plasma con una visión retrospectiva el ambiente de celebración eufórica basada en la victoria de la selección uruguaya en el Mundialito. El evento había estado condicionado por el sometimiento en las cárceles de cientos de opositores al régimen, pero incluso los uruguayos de ideología progresista que el régimen trataba de arrinconar celebraron la victoria de su selección en las calles y el estadio. No obstante, eso no fue impedimento para que en la final jugada en el estadio Centenario de Montevideo se alzaran proclamas a modo de cánticos contra la dictadura militar. Nada más terminar la final, los aficionados que asistieron al estadio celebraron el logro de su equipo representante coreando el grito de "Uruguay, Uruguay, Uruguay", pero conjuntamente añadiendo en sus coros festivos la siguiente proclama de índole política: "Se va acabar, se va acabar, la dictadura militar" (Bednarik, 2010). Manero sostiene (2013: 14) que el Mundialito se constituyó en un símbolo elocuente del fracaso de la dictadura, propiciado por la tradición democrática antecedente del país. El fútbol representó así simbólicamente la derrota sufrida antes en las urnas por el régimen.

En gran medida, la evidencia del torneo Mundialito disputado entre 1980 y 1981 en Uruguay remite a una expresión de identidad nacional unida previamente al fútbol y que trasciende a la intermediación coyuntural de la forma política en el poder y a sus 
objetivos políticos y sociales. Esa fisonomía del evento deportivo promueve un espectro aparentemente contradictorio donde los sometidos se prestan positivamente en el festejo orquestado por la autoridad que les reprime. Se ha sostenido en el marco académico, y con más vigor en la globalidad de la sociedad, que ese es el efecto del carácter narcótico del deporte asociado a la pasión patriótica. Sin embargo, la paradoja aparente no es tan contundente en la realidad empírica. El vector patriótico engendrado previamente en el fútbol posibilita que, independientemente de la manipulación promovida, represores y reprimidos se unan en un anhelo común. El Mundialito se alzó además como espacio de reivindicación política, es decir, la finalidad opuesta a la perseguida por sus promotores. Al fin y a la postre, el resurgimiento de los movimientos sociales, posteriores al evento del fútbol, fueron determinantes en la compleja transición hacia el restablecimiento de la democracia (Corbo, 2007: 30).

Con todo, el éxito del equipo uruguayo no fue monopolizado por los dirigentes que habían promocionado el campeonato como si de una herramienta de legitimación se tratase. Ciertamente los militares consiguieron aferrarse al poder hasta el año 1985, pero eso no es óbice para concluir que el acontecimiento del fútbol escenificó contrariamente a lo planeado por la dictadura, el conflicto, y la utilización del método del deporte por parte de los ciudadanos para plasmar su ansia de cambio político. La visión del manejo unidireccional y funcionalista del fútbol había sido matizada por la realidad empírica. El Mundialito quedó impregnado en la memoria uruguaya, a semejanza del Mundial de Argentina, como medio de la dictadura (Bednarik, 2010), pero también en los círculos progresistas, como el primer paso movilizador hacia la democracia (Manero, 2013: 13).

\subsection{El certamen de la Copa Confederaciones 2013 apropiado por la sociedad en Brasil}

Concerniente a Brasil, el fútbol se ha erigido en un eje primordial en su historia reciente, dado que se ha convertido en mecanismo de integración social y paralelamente en medio de consolidación de la identidad nacional, trasladando los rasgos imaginados de la identidad brasileña (Gordon y Helal, 2001: 139). Habida cuenta que la selección nacional fue percibida a partir de lo años 1950 como reflejo metonímico de la sociedad, los éxitos del equipo basados en los jugadores mestizos o negros como Garrincha o Pelé facilitaron conjuntamente el fortalecimiento de la identidad brasileña y que la diversidad étnica y racial fuese considerada como un valor añadido de la nación. A través del jogo bonito realizado en el terreno de juego por una mayoría de futbolistas mestizos que era atractivo para el espectador, el fútbol se instituyó en el deporte nacional en Brasil, y así arrinconó las visiones racistas reinantes hasta ese momento (Leite Lopes, 1997: 70-71). Después de obtener el primer Mundial de su historia en la edición de Suecia de 1958, la selección nacional se constituyó en uno de los soportes cruciales de orgullo patrio en Brasil (Knijnik, 2014: 642).

A este respecto, los gobernantes de distinta índole política trataron asiduamente de obtener réditos de la significación patriótica del fútbol en el país. Joao Goulart, a la sazón el presidente de la República de Brasil, escenificó en 1962, en el recibimiento previo al Mundial de Chile a los futbolistas brasileños, la influencia del fútbol para los ciudadanos de ese país. La segunda victoria consecutiva en el Mundial equivaldría, en palabras del presidente Goulart, a hacer olvidar a los brasileños las penurias económicas que estaban sufriendo, hasta el punto que el éxito de la selección se convertiría en más trascendental que el alimento del arroz (Solivellas Aznar, 1981: 118). De este modo, la dictadura de Medici empleó el Mundial de 1970 de México, la tercera edición ganada por el equipo nacional brasileño en la historia, a fin de adular 
a su autoritario gobierno y menguar la existencia de las torturas. Los exiliados a consecuencia del régimen habían lamentado que el éxito de la selección aseguraba a lo menos cinco años en el poder para los militares de extrema derecha en el gobierno (Alcaide Hernández, 2009: 42). El régimen de Medici utilizó el fútbol, y a su máxima estrella, Pelé, de resorte legitimador. Pero el logro en el ámbito del fútbol y su unión nítida con la expresión patriótica superó, como argumenta el historiador francés Paul Dietschy (2010: 273), también en Brasil, el uso de de la dictadura. Como expone Marcos Natali (2007: 277), en su historia reciente se pueden vislumbrar en el país las dos apropiaciones contrapuestas del acontecimiento del fútbol. A saber, el empleo de propaganda de la dictadura militar sobre la victoria en el Mundial de 1970, y el movimiento contestatario o revolucionario llamado Democracia Corinthiana en los primeros años de la década de 1980, anclado y ampliado desde el fútbol.

La iniciativa comandada por el futbolista Sócrates junto con sus compañeros del Corinthians promulgó y estableció la toma de decisiones democrática en el seno de ese club de fútbol, en una coyuntura marcada por la dictadura. La Democracia Corinthiana derivó posteriormente en la institución del movimiento a favor de las elecciones libres en Brasil, antes de diluirse en el año 1984. Jorge Knijnik (2014: 636) sugiere que el activismo político generado en la Democracia Corinthiana y comandado por un jugador influyente de la selección nacional brasileña como Sócrates, haya podido ser un germen de las protestas durante la Copa de Confederaciones acogida en Brasil en junio de 2013. El gobierno de Lula da Silva promovió activamente y logró la organización de ese evento en conjunción con el Mundial de 2014, e igualmente obtuvo la acogida de los Juegos Olímpicos de Río de Janeiro de 2016 para el país. Encuadrado en pleno auge económico, en el marco de las potencias emergentes que engloban a Brasil, Rusia, India y China, y que son denominados BRIC, Brasil suscitó la recepción de los mega eventos internacionales para impulsar su dimensión simbólica en el mundo (De Onis, 2014: 54). Pese a la mejora en los niveles de bienestar, el Partido de los Trabajadores en el poder desde el 2002, no había acometido las necesidades básicas de todos los ciudadanos en sanidad y en educación. Así las cosas, la Copa de Confederaciones de 2013 se convirtió sorpresivamente en el campo de las protestas sociales de amplio seguimiento en Brasil, que se ampliaron en las grandes urbes (De Melo Romao, 2013: 157; De Onis, 2014: 52).

La reivindicación incipiente tuvo lugar en los primeros días del mes de junio en Sao Paulo, en la antesala de la Copa de Confederaciones, en modo de protesta contra el aumento del precio del transporte colectivo en la ciudad. Así, estaba comandado por el denominado Movimiento por el Pase Libre (MPL), surgido en 2005, y que demandaba la utilidad gratuita de los transportes colectivos para el país (Tulla i Pujol et al., 2014: 2). Influido por los movimientos sociales que florecieron entonces en el mundo, a modo de lo sucedido coetáneamente en los países árabes (Muller et al., 2013: 87), a las protestas se unieron rápidamente nuevas proclamas antigubernamentales y se fueron extendiendo por más de 300 ciudades de Brasil. Cabe considerar en ese progreso la importancia de las redes sociales que se antepusieron a los medios de comunicación tradicionales. En el interior de las manifestaciones se incluyeron grupos violentos que atacaron a entidades financieras y tiendas, y dieron fuego a autobuses (De Onis, 2014: 52). Sin embargo, la reivindicación era percibida de justicia por la mayoría de la sociedad, y las cadenas de televisión estatales transformaron la condena inicial sobre las protestas por la denuncia de la represión policial acaecida (De Melo Romao, 2013: 161). El 13 de junio de 2013, en Sao Paulo, la Policía aplacó con especial vigor las protestas, con el empleo de balas de goma y gases lacrimógenos, y detuvo a más de 200 personas, incluyendo estudiantes, 
periodistas, fotógrafos y operadores de cámara, entre otros (Tulla i Pujol et al., 2014: 5). La represión y la inmediatez de las redes sociales fomentaron el auge de las marchas.

El carácter de las proclamas se diversificó más allá de la contrariedad originaria sobre la carestía del transporte público, alcanzando cada vez, una mayor magnitud social en los días previos al inicio de la Copa de Confederaciones, fijado el día 15 de junio de 2013 en la capital Brasilia. A raíz del comienzo del acontecimiento internacional, lo que hasta entonces había estado solapado o difuso en las marchas de protestas, se convirtió en la cuestión unificadora de las manifestaciones (Melo Romao, 2013: 161). A saber, la repulsa sobre la política anclada en eventos deportivos internacionales promovida por el Gobierno de Dilma Rouseff, heredero de Lula da Silva, que requería una cuantiosa inversión para la mejora de los recintos deportivos y sus accesos. La aceptación popular previa de la acogida del fútbol internacional en Brasil había tornado a ser contrariamente tomada en el espacio de las protestas, para entonces generalizadas, contra la acción de gobierno. La comparativa de las inversiones en el deporte con la situación adversa de la sanidad y la educación en el conjunto de Brasil era significante para incitar el movimiento popular (Tulla i Pujol et al., 2014: 6-7). En este sentido, a partir del 20 de junio hubo un cambio en la movilización social. El MPL, el grupo originario que reclamaba la gratuidad de los transportes colectivos, dejó de liderar el movimiento contestatario, en beneficio de los llamados Comités Populares de la Copa (CPC). Bajo su dirección, hubo manifestaciones convocadas en todas las ciudades donde la selección brasileña disputó alternativamente sus encuentros en el torneo, e igualmente en el marco de otros partidos del campeonato, si bien con menor repercusión mediática (Melo Romao, 2013: 162). Las movilizaciones fueron tomando más intensidad en el transcurso del torneo (Müller et al., 2013: 91), y todas ellas se dirimieron finalmente en enfrentamientos con la Policía. Al fin y al cabo, el efecto nacional e internacional del fútbol, en el marco de la Copa de Confederaciones, se convirtieron en un caudal impulsor de la demanda de transformación social en Brasil.

Si en la primera quincena de junio las protestas emprendidas desde la ciudad de Sao Paulo tuvieron una base eminentemente de ideología de izquierdas, coincidiendo con la Copa de Confederaciones la base de ellas se generalizó a las clases medias (Tulla i Pujol et al., 2014: 13). La oposición a las exigencias habituales impuestas por la FIFA para la organización de los eventos, y las denuncias de corrupción en el gobierno, dieron mayor sustento a las proclamas. Durante las dos semanas del torneo entre el 15 y el 30 de junio, 864.000 personas participaron en las protestas acaecidas en las seis ciudades sede del campeonato, para un total de 17 millones de brasileños mayores de 16 años en el global de las reivindicaciones sucedidas entre junio y julio de 2013 (Ibid., 9, 16). Los mensajes contestatarios tuvieron amplia continuación en los graderíos, sobreponiéndose sus pancartas y gritos a las estrictas leyes de la FIFA concernientes al absoluto monopolio en la explotación del estadio con fines comerciales (De la Cuesta, 2013). Cabe considerar asimismo, la relevancia que el seleccionador Luís Felipe Scolari y los futbolistas, Fred y Hulk, entre muchos otros, se solidarizaran en el curso de la competición con las protestas de los brasileños (La Prensa, 2013). Brasil logró posteriormente el título del campeonato de 2013 tras vencer a España por 3-0 en la final. En lo que concierne al movimiento social impulsado y mediatizado en torno al fútbol, la ausencia de un liderazgo aglutinador de las movilizaciones derivó en el debilitamiento posterior de las reivindicaciones. Al mismo tiempo, si las encuestas de opinión sobre el mandato de Dilma Rousseff realizadas en el transcurso del campeonato mostraron una perdida de apoyo de los ciudada- 
nos sobre su autoridad, las mediciones llevadas a cabo en los meses siguientes a la Copa de Confederaciones indicaron un restablecimiento de su imagen (De Melo Romao, 2013: 166). No solo eso, incluso después de la celebración del Mundial de 2014 organizado en Brasil, Dilma Rousseff revalidó su mandato en la segunda vuelta de las elecciones presidenciales con un el $51,63 \%$ de los votos, frente al $48,37 \%$ de los sufragios obtenidos por su rival para la presidencia del país, Aécio Neves.

Como consideración final de la Copa de Confederaciones acogida recientemente en Brasil, cabe constatar la acaparación del binomio del fútbol y nación hecha tanto por el poder político como por parte de la sociedad civil. La influencia del fútbol magnificó las protestas iniciales en contra de la gestión ineficaz del Partido de los Trabajadores en la socialización de los réditos del crecimiento económico en el país emergente. Tras el fin de la Copa de Confederaciones y en la previa del Mundial, el movimiento perdió el auge originario, y paralelamente, el Partido de los Trabajadores y la presidenta Rousseff pudieron renovar el mandato, pese a las denuncias fundamentadas de corrupción en su seno. En gran medida, la concatenación de acontecimientos deportivos en Brasil resultó ser un vector idóneo a fin de contrariar el orden establecido y plasmar el descontento de la sociedad brasileña. El país cuyo orgullo patrio se sustentó nítidamente en el fútbol y en la selección nacional durante el siglo XX, se apropió de su alcance para demandar una transformación en la conducción de la nación en el siglo XXI. En este caso, Brasil antepuso la revolución en el deporte al patriotismo simbólico gestado en su celebración.

\section{Conclusiones}

El Mundial de Argentina en 1978, el Mundialito de Uruguay en 1980 y la Copa de Confederaciones de Brasil en el año 2013 sugieren una evolución contemporánea en la apropiación de la sociedad civil del binomio del fútbol y de la dimensión nacional en América del Sur, y que es extrapolable al ámbito internacional. Por una parte, esa unión originaria propició en Argentina 1978 el anhelo común de represores y reprimidos por la victoria de su selección, y el sometimiento de los derechos humanos fundamentales al ideario simbólico del estado-nación. Durante el Mundialito de Uruguay del año 1980, los uruguayos que estaban regidos también por un régimen coercitivo, emplearon finalmente el campeonato internacional para demandar el final de la dictadura, al mismo tiempo que celebraban el triunfo de su representación nacional. Por último, en el caso más reciente de la Copa de Confederaciones de 2013 en Brasil, una notoria parte de la sociedad civil organizada sobrepuso las proclamas sociales en el marco del fútbol a la euforia nacional conducida a través de la representación nacional.

Sin obviar la complejidad de equiparar realidades diferenciadas, el acercamiento histórico y evolutivo posibilita vislumbrar la progresión sucedida en lo que a la apropiación del evento del fútbol por la iniciativa independiente de la sociedad civil se refiere. En América del Sur esta tendencia confluye temporalmente con la transición acaecida desde el autoritarismo al liberalismo y a la posterior institución de gobiernos social demócratas en numerosas sociedades de la región (Lanzaro, 2013: 251). En esta coyuntura, el fútbol es usado cada vez con más pujanza en su vertiente transformadora, sin menoscabo alguno, o en confluencia, con su significación patriótica y comunitaria. El deporte refleja la fortaleza de los movimientos de base popular en el mundo, y remite a desterrar la visión única de manejo funcionalista e unidireccional del fútbol, de arriba hacia abajo. Es, efectivamente, un espacio donde es reflejada 
la lucha, dispar eso sí, de diferentes ámbitos de poder dentro de las sociedades contemporáneas.

Los casos estudiados muestran que ni la significación comunitaria del deporte, ni su utilización, son exclusividad del poder instituido en los estados, sino que pertenecen en la misma medida a la atribución distintiva llevada a cabo por los ciudadanos. Ésta se puede materializar en diferentes facetas, incluyendo la motivación transformadora o de resistencia. Pese a todo, el fútbol y el deporte perviven en el imaginario colectivo de las sociedades como medio de manipulación de la élite política o económica dirigente. De esa visión subyace notoriamente la percepción hegemónica persistente de las relaciones de poder dentro de las sociedades del siglo XXI. Cabe destacar finalmente que tampoco la utilidad transformadora del fútbol es por si misma concluyente, como lo demuestra la victoria de Dilma Rousseff en Brasil en las elecciones presidenciales posteriores a las protestas sucedidas durante la Copa de Confederaciones 2013 y en el previo al Mundial de 2014. Así, la reflexión en las ciencias sociales requiere relativizar el sentido de los acontecimientos para poder atisbar la realidad conflictual con el mayor rigor científico.

\section{Bibliografía}

Agüero Wagner, L. (2010). "Las semifinales del Mundial y el cura de la eliminación". Diario Siglo XXI. 6 de julio. http://www.diariosigloxxi.com/texto-diario/mostrar/57740\#. PIT2Gh3ojFOKicV

Alabarces, P. y Rodríguez, M. G. (1999). "Football and Fatherland: The crisis of national representation in Argentinian soccer". Culture, Sport, Society, vol. 2, n 3, pp. 118-133. DOI: $10.1080 / 14610989908721849$

Alabarces, P. (1999). "Post-Modern Times: Identities and Violence in Argentine football". En G. Armstrong, y R. Giulianotti. (Eds), Football Cultures and Identities (pp. 77-85). Londres: Macmillan Press.

Alcaide Hernández, F. (2009). Fútbol: Fenómeno de fenómenos. Barcelona: LID Editorial Empresarial.

Arbena, J. L. (1996). "Nationalism and Sport in Latin America, 1850-1990: The Paradox of Promoting and Performing 'European' Sports”. En J.A. Mangan. (Ed.), Tribal Identities: Nationalism, Europe, Sport (pp. 220-238). Londres: Frank Cass.

Archetti, E. P. (2006). "Argentina 1978: Military Nationalism, Football Essentialism, and Moral Ambivalence". En A. Tomlinson, C. Young. (Eds), National Identity and Global Sports Events: Culture, Politics, and Spectacle in the Olympics and the Football World Cup (pp. 133-147). Nueva York: State University of New York Press.

Archetti, E.P. (1994). "Masculinity and football: The formation of national identity in Argentina". En R. Giulianotti, y J. Williams. (Eds), Game Without Frontiers: Football, identity and modernity (pp. 225-243). Reino Unido: Arena.

Auge, M. (1999). “¿Un deporte o un ritual?”. En S. Segurola. (Ed.), Fútbol y pasiones políticas (pp. 55-66). Madrid: Debate.

Beck, P. J. (2001). "For World Footballing Honours: England versus Italy, 1933, 1934 and 1939". En J.A. Mangan. (Ed.), Europe, Sport, World: Shaping Global Societies (pp. 245-266). Londres: Frank Cass. 
Bednarik, S. (2010). Mundialito. Documental producido por Coral Films y V2 Cinema. Uruguay y Brasil. Duración: 75 minutos.

Billings, A. C. (2008). Olympic Media: Inside the biggest show on television. Londres: Routledge.

Boniface, P. (2002). La Terre est ronde comme un ballon: Géopolitique du football. Paris: Seuil.

Boyle, R. y Haynes, R. (2009). Power play: Sport, the Media and Popular Culture. Londres: Edinburgh University Press.

Brohm, J.-M. y Perelman, M. (2006). Le football, une peste émotionnelle: La barbarie des stades. Paris: Gallimard.

Bromberger, C. (1998). La bagatelle la plus sérieuse du monde. Paris : Bayard Éditions.

Bromberger, C. (1995). Le match de football. Ethnologie d'une passion partisane à Marseille, Naples et Turin. Paris: Éditions de la Maison des sciences de l'homme.

Corbo, D. (2007). "La transición de la dictadura a la democracia en Uruguay. Perspectiva comparada sobre los modelos de salida política en el Cono Sur de América Latina". Humanidades: Revista de la Universidad de Montevideo, vol. VII, nº 1, pp. 23-47.

Darbon, S. (2008). Diffusion des sports et impérialisme anglo-saxon: De l'histoire événementielle à l'anthropologie. Paris: Éditions Maison des Sciences de l'Homme.

De la Cuesta, N. (2013). Las protestas en Brasil ponen en riesgo la Copa Confederaciones. Diario El Mundo. 21 de junio. http://www.elmundo.es/elmundodeporte/2013/06/21/futbol/1371810100.html

De Melo Romao, W. (2013). "\#naovaitercopa: manifestações, Copa do Mundo e as eleições de 2014". Revista de Discentes de Ciência Política da UFSCAR, vol.1, $\mathrm{n}^{\circ} 2$, pp. 152-167.

De Onis, J. (2014). "Brazil's troubles: World Cup runneth over". World Affairs, pp. 51-57.

Dietschy, P. (2010). Histoire du football. Paris: Perrin.

Duke, V. y Crolley, L. (2001). "Fútbol, Politicians and the People: Populism and Politics in Argentina". International Journal of the History of Sport, vol.18, $\mathrm{n}^{\circ} 3$, pp. 93-116. DOI: 10.1080/714001587

Dunning, E. (1992). "La dinámica del deporte moderno". En N. Elias y E. Dunning. (Eds), Deporte y Ocio en el proceso de la civilización (pp. 247-269). Madrid: Fondo de Cultura Económica.

Eisenberg, C.; Lanfranchi, P., Mason, T. y Wahl, A. (2004). FIFA 1904-2004: Un siglo de fútbol. Madrid: Pearson Educación.

Fernandez Moores, E. (2006). Sudamérica: de la gloria a cantera de Europa. Vanguardia Dossier, $n^{\circ} 20$, pp. 67-72.

Galeano, E. (2003). El Fútbol a sol y sombra. Madrid: Siglo XXI.

Goldberg, D. T. (2002). The Racial state. Malden (EEUU): Blackwell Publishing.

Gordon, C. y Helal, R. (2001). "The Crisis of Brazilian Football: Perspectives for the Twenty-First Century". International Journal of the History of Sport, vol. 18, $n^{\circ} 3$, pp. 139158. DOI: $10.1080 / 714001590$ 
Holt, R. (2002). "El Ministerio de Asuntos Exteriores y la Asociación de Fútbol: Deporte británico y apaciguamiento (1935-1938)". En T. González Aja. (Ed.), Sport y autoritarismos. La utilización del deporte por el comunismo y el fascismo (pp. 79-102). Madrid: Alianza Editorial.

Jarvie, G. y Joseph Maguire. (1994). Sport and Leisure in Social Thought. Londres: Routledge.

Kapuściński, R. (1992). La guerra del fútbol y otros reportajes. Barcelona: Editorial Anagrama.

Knijnik, J. (2014). "Playing for freedom: Sócrates, futebol-arte and democratic struggle in Brazil". Soccer \& Society, vol. 15, $\mathrm{n}^{\circ}$ 5, pp. 635-654. DOI: $10.1080 / 14660970.2014 .912014$

La Prensa. (2013). "Brasil: Jugadores de la selección apoyan las protestas en su país". 18 de junio. http://laprensa.pe/actualidad/noticia-brasil-jugadores-seleccion-apoyan- protestassu-pais-8667

Lanzaro, J. (2013). "Continuidad y cambios en una vieja democracia de partidos-Uruguay (1910-2010)". Opiniao pública, vol. 19, n² 2, pp. 235-269. http://dx.doi.org/10.1590/ S0104-62762013000200001

Leite Lopes, J. S. (1997). "Successes and Contradictions in 'Multiracial' Brazilian Football”. En G. Armstrong, y R. Giulianotti. (Eds), Entering the Field: New Perspectives on World Football (pp. 53-86). Oxford: Berg.

MacClancy, J. (1996). Sport, Identity and Ethnicity. Oxford: Berg.

Manero, C.D. (2013). "Fútbol y dictadura en Uruguay: El Mundialito desde Bourdieu y Elías". Revista de ALESDE, vol. 3, n² 2, pp. 4-14.

Manzenreiter, W. (2006). "Sports spectacles, uniformities and the search for identity in late modern Japan”. En J. Horne, y W. Manzenreiter. (Eds), Sports Mega-Events: Social Scientific Analyses of a Global Phenomenon (pp. 144-159). Malden: Blackwell.

Martín Barbero, J. (1980). "Retos a la investigación de comunicación en América Latina". Humánitas, Portal Temático en Humanidades.

Mattelart, A. y Mattelart, M. (1987). Pensar sobre los medios. Madrid: Fundesco.

Muller, I.D., Luiz de Oliveira Junior, L., Ferandes Feltes, A., y Roese Sanfelice, G. (2013). "Manifestos sociais e Copa das Confederações na cobertura da folha de São Paulo". Motrivivência, vol. XXV, $\mathrm{n}^{\circ}$ 41, pp. 85-100. http://dx.doi. org/10.5007/2175-8042.2013v25n41p85

Natali, M. (2007). "The Realm of the Possible: Remembering Brazilian Futebol". Soccer \& Society, vol.8, n 2/3, pp. 267-282. DOI: 10.1080/14660970701224491

Rein, R. y Davidi, E. (2009). "Sport, Politics and Exile: Protests in Israel during the World Cup (Argentina, 1978)". The International Journal of the History of Sport, vol. 26, $\mathrm{n}^{\circ} 5$, pp. 673-692. DOI: 10.1080/09523360902722666

Roldán, D.P. (2007). "La espontaneidad regulada: Fútbol, autoritarismo y nación en Argentina 78. Una mirada desde los márgenes". Prohistoria, vol. 11, pp. 125-147.

Rouquié, A. (1971). "Honduras-El Salvador. La guerre de cent heures: un cas de «désintégration» regionales". Revue française de science politique, vol.21, nº 6, pp. 1290-1316. 
Salvador, J. L. (2004). El deporte en occidente: Historia, cultura y política. Madrid: Ediciones Cátedra.

San Román, G. (2005). "La garra charrúa: fútbol, indios e identidad en el Uruguay contemporáneo". Bulletin Hispanique, vol. 107, n² 2, pp. 633-655.

Smith, B. L. (2002). "The Argentinian Junta and the Press in the Run-up to the 1978 World Cup". Soccer \& Society, vol. 3, nº 1, pp. 69-78. DOI: 10.1080/714004869

Solivellas Aznar, M. (1981). Futbol, Ideología y política: Los campeonatos del mundo. (Tesis de obtención del grado de licencia bajo la dirección de Borja de Riquer). Barcelona, Universitat Autònoma de Barcelona.

Sonntag, A. (2010). "Sentiments mitigés-identités multiples. Le football contemporain à l'ère de la globalisation". En U. Pfeil. (Ed.), Football et identité en France et Allemagne (pp. 209-234). Lille (Francia): Presses Universitaires du Septentrion.

Tulla i Pujol, A.; Goulart Rocha, F. y Dos Santos Sampaio, F. (2014). Manifestações populares no Brasil atual: Sociedade civil em rede e reivindicações sobre o poder político. XIII Coloquio Internacional de Geocrítica: El control del espacio y los espacios de control, Barcelona, 5-10 de Mayo de 2014.

Wahl, A. (1997). Historia del fútbol, del juego al deporte. Barcelona: Ediciones B.

Yaffé, J. (2012). "La dictadura uruguaya (1973-1985): nuevas perspectivas de investigación e interpretación historiográfica". Estudos Ibero-Americanos, vol. 38, n¹, pp. 13-26.

Yonnet, P. (2008). "Composants de l'identité, mécanismes de l'identification". En J.M. De Waele, y A. Husting (Eds), Football et identités (pp. 19-31). Bruselas: Éditions de l'Université de Bruxelles. 